\title{
Geodesic stability for KS Black hole in Hořava-Lifshitz gravity via Lyapunov exponents
}

\author{
MR.Setare \\ Department of Science, Payame Noor University, Bijar, Iran* \\ D.Momeni \\ Department of Physics,faculty of Basic Sciences, \\ Tarbiat Moalem University,Karaj, Iran
}

\begin{abstract}
By computing the Lyapunov exponent, which is the inverse of the instability time scale associated with this geodesic motion we show that there is two region of space which in both of them the equatorial timelike geodesics are stable via Lyapunov measure of stability.

PACS numbers: $04.70 . \mathrm{Bw}, 04.50 . \mathrm{Gh}$
\end{abstract}

\section{MATHEMATICAL PRELIMINARIES:LYAPUNOV EXPONENTS}

Let us consider a trajectory (in phase space) described by a certain evolution. The Lyapunov exponents (also known as characteristic exponents) associated with a trajectory are essentially a measure of the average rates of expansion and contraction of trajectories surrounding it. They are asymptotic quantities, defined locally in state space, and describe the exponential rate at which a perturbation to a trajectory of a system grows or decays with time at a certain location in the state space[1]. Analysis conducted with Lyapunov exponents are called Lyapunov stability analysis. They are useful in characterizing the asymptotic state of an evolution (attractors in dissipative systems)[2]. Using Lyapunov exponents, we can distinguish among fixed points, periodic motions, quasiperiodic motions, and chaotic motions[3].

\section{Concept of Lyapunov Exponents}

We begin by defining Lyapunov exponents for a given system of equations. Let $X(t)$ such that $X(t=0)=X_{0}$ represent a trajectory of the system governed by the following n-dimensional autonomous system:

$$
\dot{x}=F(x ; M)
$$

where the vector $x$ is made up of $n$ state variables, the vector function $\mathrm{F}$ describes the nonlinear evolution of the system, and $\mathrm{M}$ represents a vector of control parameters. Denoting the perturbation provided to $X(t)$ by $y(t)$ and assuming it to be small, we obtain an equation after linearization in the disturbance terms. The perturbation is governed by

$$
\dot{y}=A y
$$


where, in general, $A=D_{x} F[x(t) ; M]$ is an $n \times n$ matrix with time dependent coefficients. If we consider an initial deviation $y(O)$, its evolution is described by

$$
y(t)=\phi(t) y(0)
$$

where $\phi(t)$ is the fundamental (transition) matrix solution of (3) associated with the trajectory $X(t)$. The eigenvalues of A provide information about the stability of the associated fixed point.

The procedure used to determine Lyapunov exponents can be considered to be a generalization of linear stability analyses. An interesting and detailed discussion on the relationship between linear stability analysis and Lyapunov stability analysis can be found in the paper of Goldhirsch, Sulem, and Orszag [4]. They argue that the Lyapunov exponents are global quantities associated For an appropriately chosen $y(0)$ in $(3)$, the rate of exponential expansion or contraction in the direction of $y(0)$ on the trajectory passing through $X_{0}$ is given by

$$
\lambda_{j}=\lim _{t \rightarrow \infty} \frac{1}{t} \log \frac{\|y(t)\|}{\|y(0)\|}
$$

where the symbol $\|$ denotes a vector norm . The asymptotic quantity $\lambda_{j}$ is called the Lyapunov exponent. We have $\mathrm{n}$ Lyapunov exponents associated with an $\mathrm{n}$-dimensional autonomous system. It can be shown that if the trajectory $X(t)$ corresponds to a motion other than a fixed point, then one of the $\lambda_{j}$ is always zero . Following Lyapunov [5], the fundamental matrix $\phi(t)$ is called regular if

$$
\lim _{t \rightarrow \infty} \frac{1}{t} \log \operatorname{det}(\phi(t))
$$

exists and is finite. If $\phi(t)$ is regular, then, according to a theorem, the asymptotic quantity defined in (4) exists and is finite for any initial deviation $y(0)$ belonging to the $\mathrm{n}$-dimensional space.

\section{STABILITY OF CIRCULAR ORBITS VIA LYAPUNOV EXPONENTS}

As was shown by Cardoso et al[6] for all spherically symmetric spacetimes, in a geometrical optics approximation, QNMs can be interpreted as particles trapped at unstable circular null geodesics and slowly leaking out. The leaking time scale is given by the principal Lyapunov exponent, which one can obtain a fairly simple expression, in terms of the second derivative of the effective radial potential for geodesic motion. As was stated by Cardoso et al, this deep, intuitive approach to the QNMs and it's relation to the circular null geodesics is valid for all asymptotically flat, spherically symmetric black-hole spacetimes. The most important note is that the powerfull formalism which is presented in [5] is completely independent from the form of the action, i.e there is no difference between treating a stationary, asymptotically flat, static spacetime from a higher order gravity as $f(R)$ or HL and the usual higher dimensional (or 4-dim) BHs in GR.Thus with have no worrying about the validity of their method we can use it and following all the steps of the [6]. Without no loss of generality we can restrict ourselves to a simple kind of problems includes circular orbits in stationary spherically symmetric spacetimes and equatorial circular orbits in stationary spacetim .This 
case could be described by a two-dimensional phase space which for our geodesics analysis may be described by

$$
X(t)=\left(p_{r}, r\right)
$$

Linearizing the equations of motion about orbits of constant $r$, the principal Lyapunov exponents can be expressed as

$$
\lambda=\sqrt{\frac{V_{r}^{\prime \prime}}{2 \dot{t}^{2}}}
$$

Where $V_{r}=\dot{r}^{2}$ and a dot denotes the derivative with respect to the proper time $\tau$. Remember to mind that For circular geodesics

$$
V_{r}=V_{r}^{\prime}=0
$$

\section{KS BLACK HOLE SOLUTION IN HL THEORY}

There are different versions of the HL theory.As a geometrical point of view all these versions Follow from the ADM decomposition of the metric [7], the fundamental objects of interest are the fields $N(t, x), N_{i}(t, x), g_{i j}(t, x)$ corresponding to the lapse, shift and spatial metric of the ADM decomposition. In the $(3+1)$-dimensional ADM formalism, where the metric can be written as

$$
d s^{2}=-N^{2} d t^{2}+g_{i j}\left(d x^{i}+N^{i} d t\right)\left(d x^{j}+N^{j} d t\right)
$$

and for a spacelike hypersurface with a fixed time, its extrinsic curvature $K_{i j}$ is

$$
K_{i j}=\frac{1}{2 N}\left(\dot{g_{i j}}-\nabla_{i} N_{j}-\nabla_{j} N_{i}\right)
$$

where a dot denotes a derivative with respect to $t$ and covariant derivatives defined with respect to the spatial metric $g_{i j}$, the action of Horava-Lifshitz theory for $z=3$ is

$$
S=\int_{M} d t d^{3} x \sqrt{g} N\left(\mathcal{L}_{K}-\mathcal{L}_{V}\right)
$$

we define the space-covariant derivative on a covector $v_{i}$ as $\nabla_{i} v_{j} \equiv \partial_{i} v_{j}-\Gamma_{i j}^{l} v_{l}$ where $\Gamma_{i j}^{l}$ is the spatial Christoffel symbol. $g$ is the determinant of the 3 -metric and $N=N(t)$ is a dimensionless homogeneous gauge field. The kinetic term is

$$
\mathcal{L}_{K}=\frac{2}{\kappa^{2}} \mathcal{O}_{K}=\frac{2}{\kappa^{2}}\left(K_{i j} K^{i j}-\lambda K^{2}\right)
$$

Here $N_{i}$ is a gauge field with scaling dimension $\left[N_{i}\right]=2$.

The potential term $\mathcal{L}_{V}$ of the $(3+1)$-dimensional theory is determined by the principle of detailed balance [8], requiring $\mathcal{L}_{V}$ to follow, in a precise way, from the gradient flow generated by a 3 -dimensional action $W_{g}$. This principle was applied to gravity with the result that 
the number of possible terms in $\mathcal{L}_{V}$ are drastically reduced with respect to the broad choice available in an potential is

$$
\mathcal{L}_{V}=\alpha_{6} C_{i j} C^{i j}-\alpha_{5} \epsilon_{l}^{i j} R_{i m} \nabla_{j} R^{m l}+\alpha_{4}\left[R_{i j} R^{i j}-\frac{4 \lambda-1}{4(3 \lambda-1)} R^{2}\right]+\alpha_{2}\left(R-3 \Lambda_{W}\right)
$$

Where in it $C_{i j}$ is the Cotton tensor [9]which is defined as,

$$
C^{i j}=\epsilon^{k l(i} \nabla_{k} R_{l}^{j)}
$$

The kinetic term could be rewritten in terms of the de Witt metric as:

$$
\mathcal{L}_{K}=\frac{2}{\kappa^{2}} K_{i j} G^{i j k l} K_{k l}
$$

Where we have introduced the de Witt metric

$$
G^{i j k l}=\frac{1}{2}\left(g^{i k} g^{j l}+g^{i l} g^{j k}\right)-\lambda g^{i j} g^{k l}
$$

Inspired by methods used in quantum critical systems and non equilibrium critical phenomena, Horava restricts the large class of possible potentials using the principle of detailed balance outlined above. This requires that the potential term takes the form

$$
\mathcal{L}_{V}=\frac{\kappa^{2}}{8} E^{i j} G_{i j k l} E^{k l}
$$

Note that by constructing $E^{i j}$ as a functional derivative it automatically transverse within the foliation slice, $\nabla_{i} E^{i j}=0$. The equations of motion were obtained in [10]. KS BH is a static spherically symmetric solution for HL theory which contains 2 parameter, one mass like parameter $m$ and a parameter which controls the escape from a naked singularity $\omega$ and satisfies [11]

$$
\omega m^{2} \geq \frac{1}{2}
$$

In the usual spherical coordinates $(t, r, \theta, \phi)$ and in the Schwarzschild's gauge the metric reads:

$$
d s^{2}=\operatorname{diag}\left(+f,-\frac{1}{f},-r^{2} \Sigma_{2}\right)
$$

where in it the metric gauge function is

$$
f=1+\omega r^{2}-\sqrt{\omega^{2} r^{4}+4 m \omega r}
$$

and is $\Sigma_{2}$ the surface element on a unit 2- sphere. As motivated by Sekiwa "it is obvious that $1 / 2 \omega$ is equivalent to $Q^{2}$ and this means that we could view $1 / 2 \omega$ as a charge in some degree"[12]. Thus The outer and inner event horizon can be compared with the outer and inner event horizon of Reissner-Nordstrom black hole [13]. Essentially as claimed by the founders of the $\mathrm{KS}$, this solution "represents the analog of the Schwarzschild solution of GR". 


\section{CIRCULAR ORBITS}

The best treatment of the geodesics equations is due to Chandrasekhar [14].For a simple typical form of a spherically symmetric metric in Schwarzschild gauge

$$
d s^{2}=\operatorname{diag}\left(+f(r),-\frac{1}{g(r)},-r^{2} \Sigma_{2}\right)
$$

one can obtain the following expression for the potential function:

$$
V_{r}=g(r)\left(\frac{E^{2}}{f(r)}-\frac{L^{2}}{r^{2}}-\delta_{1}\right)
$$

Where in it the $E, L$ respectably can be interpreted as the Energy,angular momentum of the test particle within the circular orbits in similar to the classical mechanics and $\delta_{1}=1,0$ for timelike and null geodesics, respectively.For KS BH (8) the relation (11) convert to:

$$
V_{r}=E^{2}-\frac{L^{2} f(r)}{r^{2}}-\delta_{1} f(r)
$$

\section{TIMELIKE GEODESICS}

For circular orbits we know that both the potential term and the first derivates of it must be vanish which leads us to the next expression for the second derivative of the potential

$$
V_{r}^{\prime \prime}=2\left[\frac{-3 f f^{\prime} / r+2 f^{\prime 2}-f f^{\prime \prime}}{2 f-r f^{\prime}}\right]
$$

thus using (7) the Lyapunov exponent at the circular timelike geodesics is

$$
\lambda=\frac{1}{2} \sqrt{\left(2 f-r f^{\prime}\right) V_{r}^{\prime \prime}}
$$

Since the energy must be real, we require

$$
\frac{\partial \log (f(r))}{\partial \log (r)}<2
$$

this inequality leads to the next bound for the radius of the timelike circular orbit ${ }^{1}$

$$
\omega^{2} r^{3}-9 m^{2} \omega^{2} r+4 m \omega>0
$$

This is a cubic algebraic equation. We review the general solution for a cubic eq.Let the general cubic eq be

$$
x^{3}+p x+q=0
$$

To have three distinct real root(the case which is happen in KS BH) we define

$$
\Delta=4 p^{3}+27 q^{2}
$$

\footnotetext{
${ }^{1}$ Remember that in KS BH we must have $m^{2} \omega \geq \frac{1}{2}$.
} 
If $\Delta<0$ then these three roots can be obtained by solving a simple trigeometric

$$
\cos (3 \theta)=\cos (\alpha)
$$

where in it

$$
\begin{array}{r}
\cos (\alpha)=-\frac{4 q}{A^{3}} \\
A^{2}=-\frac{4 p}{3} \\
x=A \cos (\theta)
\end{array}
$$

for radial eq (16) we have

$$
\Delta=-108\left(\frac{m}{\omega}\right)^{2} \delta \leq-297\left(\frac{m}{\omega}\right)^{2}
$$

where in it

$$
\delta=27 m^{6} \omega^{6}-4 m^{2} \omega^{4} \geq 2.75
$$

finally we can write the next expressions for all roots of the (16)

$$
r_{n}=2 \sqrt{3} m \cos \left(\frac{2 n \pi}{3} \pm \frac{\alpha}{3}\right), n=0,1,2
$$

Also we know that the KS solution has a physically accessible horizon located at

$$
r=h=m+\sqrt{m^{2}-Q^{2}}
$$

The KS solution is valid only for such region of the space where $r \geq h$. We must determine that which of these three distinct real values $r_{i}, i=0,1,2$ belongs to this interval.Indeed we must have

$$
h<r_{n} \leq 2 \sqrt{3} m
$$

(Which is satisfied automatically)

Also the negativity of the energy impose that for timelike geodesics we must restricted ourselves to

$$
\left(r_{0}<r<r_{1}\right) \curlyvee\left(r>r_{2}\right)
$$

This bound for the radial coordinates which arisen from the reality of the Energy has an essential role for investigating the stability via the relation (14) for the Lyapunov exponent.If we want to have a stable motion the exponent $\lambda$ must be imaginary and have a negative real part and unstablee unless.For instability we must have

$$
V_{r}^{\prime \prime}>0
$$

The dominator of it must be checked. That is we must check that when $r$ belongs to the (26) the next inequality satisfied or not?

$$
-3 f f^{\prime} / r+2 f^{\prime 2}-f f^{\prime \prime}>0
$$

the opposite sign indicates on astable one. A very simple and straightforward calculation show that for all values of the $r$ belong to the (26) we have

$$
V_{r}^{\prime \prime}<0
$$

Thus the time like geodesics in $\mathrm{KS} \mathrm{BH}$ are stable. 


\section{CONCLUSION}

HL theory brings some important new features from the GR to the higher dimensional lagrangian and it's role in construction a non relativistic candidate for quantum gravity. The Lyapunov exponents is a very important and powerful method to stability any dynamical system both in classical mechanics and also in quantum treatment of complex systems.I $\mathrm{n}$ this short letter after a brief review of the Lyapunov method in mathematical physics, following the method which have been described by Cardoso et al[5] for 4 and 5 dimensional $\mathrm{BHs}$ in GR,we applied a similar method to the a new type of the BH which indeed are a formal IR limit of the HL theory and show that the timelike geodesics are stable under small perturbations.As Cardoso et al show that for a equatorial circular timelike geodesics in a Myers-Perry black-hole background are unstable .But we show that in the context of the HL theory these geodesics are stable. Thus we can argued that the nonlinear terms are successful to pass from the instabilities.

* Electronic address: rezakord@mail.ipm.ac.ir

1 Michael Wilkinson,"Title: Lyapunov exponent for small particles in smooth one-dimensional flows", arXiv:0911.2917

J. Vanneste,"Estimating generalised Lyapunov exponents for products of random matrices", arXiv:0911.2161

Rudolf A Roemer, Hermann Schulz-Baldes," The random phase property and the Lyapunov spectrum for disordered multi-channel systems", arXiv:0910.5808

Parthapratim Biswas, H. Shimoyama, L.R.Mead,"Title: Lyapunov exponent and natural invariant density determination of chaotic maps: An iterative maximum entropy ansatz" arXiv:0910.4561]

[1] Young, L.-S. (1982). Dimension, entropy, and Lyapunov exponents, Erg. Theory Dyn. Syst. 2, 109-124. 598

Young, L . 4 . (1983).Entropy, Lyapunov exponents, and Hausdorff dimension in differentiable dynamic systems, IEEE Trans. Circuit Syst. 8, 599-607. 538

[2] Zeeman, E. C. (1982). Bifurcation and catastrophe theory, in Papers in Algebra, Analysis, and Statistics, R. Lidl, ed., American Mathematical Society, Providence, Rhode Island, 207-272. 68

Tabor, M. (1989). Chaos and Integrability in Nonlinear Dynamics, Wiley, New York.

Spirig, F. (1983). Sequence of bifurcations in a three-dimensional system near a critical point, J. Appl. Math. Phys. (ZAMP) 34, 259- 276. 233

Stavans, J., F. Heslot, and A. Libchaber (1985). Fixed winding number and the quasiperiodic route to chaos in a convective fluid, Phys. Rev. Lett. 55, 596-599. 248, 323

Sommerer, J. C., W. L. Ditto, C. Grebogi, E. Ott, and M. L. Spano (1991). Experimental confirmation of the theory for critical exponents of crises, Phys. Lett. A 153, 105-109.

[3] I. Goldhirsch, P.L. Sulem and S. Orszag, Physica D 27, 311 (1987)

[4] Lyapunov, A. M. (1992), The general problem of the stability of motion, London: Taylor and Francis, ISBN 978-0748400621

[5] Vitor Cardoso,Alex S. Miranda,Emanuele Berti,Helvi Witek,Vilson T. Zanchin,Phys. Rev. D $79,064016(2009)$ 
[6] Richard Arnowitt Stanley Deser Charles W. Misner,"Republication of: The dynamics of general relativity",Gen Relativ Gravit (2008) 40:19972027 DOI 10.1007/s10714-008-0661-1

[7] P. Hořava JHEP03(2009)020 doi: 10.1088/1126-6708/2009/03/020

[8] P. Hořava, Phys. Rev. D 79084008 (2009) arXiv:0901.3775 [hep-th]]. P. Hořava, Phys.Rev.Lett. 102, 161301 (2009) arXiv:0902.3657 [hep-th]].

[9] E. Kiritsis and G. Kofinas, Hořava-Lifshitz cosmology,Nucl.Phys.B821:467-480,2009 DOI: 10.1016/j.nuclphysb.2009.05.005 arXiv:0904.1334 [hep-th].

[10] A. Kehagias and K. Sfetsos, Phys Lett B 678, 123 (2009) arXiv:0905.0477 [hep-th]]

[11] M. Wang, J. Jing, C. Ding, and S. Chen , "First laws of thermodynamics in IR Modified Hořava-Lifshitz gravity," arXiv:0912.4832 [gr-qc]].

[12] Reissner, H.(1916). Uber die Eigengravitation des elektrischen Feldes nach der Einsteinschen Theorie.Ann. Phys. (Germany) 50, 106.See 15.4, 21.1.

[13] S. Chandrasekhar, The Mathematical Theory of Black Holes (Oxford University Press, New York, 1983). 